\title{
Diameter-driven crossover in resistive behaviour of heavily doped self-seeded germanium nanowires
}

\author{
Stephen Connaughton ${ }^{1}$, Maria Koleśnik-Gray ${ }^{2}$, Richard Hobbs ${ }^{3}$, Olan Lotty ${ }^{3}$, \\ Justin D. Holmes ${ }^{3}$ and Vojislav Krstić ${ }^{* 1,2}$
}

\section{Letter}

Address:

${ }^{1}$ School of Physics, AMBER@CRANN, Trinity College Dublin, College

Green, Dublin 2, Republic of Ireland, ${ }^{2}$ Chair for Applied Physics,

Department of Physics, Friedrich-Alexander-University

Erlangen-Nürnberg (FAU), Staudtstraße 7, 91058 Erlangen, Germany

and ${ }^{3}$ Materials Chemistry \& Analysis Group, Department of

Chemistry, University College Cork, Cork, Republic of Ireland

Email:

Vojislav Krstić - vojislav.krstic@fau.de

* Corresponding author

Keywords:

diameter-dependence; germanium; nanowire; resistivity; self-seeded
Beilstein J. Nanotechnol. 2016, 7, 1284-1288.

doi:10.3762/bjnano.7.119

Received: 15 June 2016

Accepted: 25 August 2016

Published: 13 September 2016

Associate Editor: J. M. van Ruitenbeek

(C) 2016 Connaughton et al.; licensee Beilstein-Institut. License and terms: see end of document.

\begin{abstract}
The dependence of the resistivity with changing diameter of heavily-doped self-seeded germanium nanowires was studied for the diameter range 40 to $11 \mathrm{~nm}$. The experimental data reveal an initial strong reduction of the resistivity with diameter decrease. At about $20 \mathrm{~nm}$ a region of slowly varying resistivity emerges with a peak feature around $14 \mathrm{~nm}$. For diameters above $20 \mathrm{~nm}$, nanowires were found to be describable by classical means. For smaller diameters a quantum-based approach was required where we employed the 1D Kubo-Greenwood framework and also revealed the dominant charge carriers to be heavy holes. For both regimes the theoretical results and experimental data agree qualitatively well assuming a spatial spreading of the free holes towards the nanowire centre upon diameter reduction.
\end{abstract}

\section{Findings}

Semiconducting nanowires are in the focus of research due to their potential applications in electronics and optics [1-9]. Germanium nanowires (Ge NWs) are of particular interest as they provide the prospect for quantum-related phenomena associated with one-dimensional (1D) confinement already at diameters of tens of nm [10], or determining their electronic properties by surface doping [11].
Among different synthetic routes for obtaining Ge NWs $[12,13]$, the novel self-seeding mechanism is of special interest $[14,15]$. The main advantage of this method is the elimination of dopant incorporation from the metal nanoparticle catalysts [11-13]. It was demonstrated that by selecting the synthesis conditions, the degree of surface-doping in the NW can be controlled [11]. Even heavy doping close to or at the degeneracy 
level can be achieved, rendering the semiconducting NW quasimetallic [11]. Approaching degenerate doping, therefore, provides opportunity to produce NWs adequate for both nanoscaled semiconductor conduction-channel and source- and drain-components. Ultimately, these two doping states could be realised within the same NW in different sections. In perspective of the continued successful miniaturisation of electronic devices it is therefore essential to elucidate the diameter dependence of the resistivity of heavily/degenerately doped selfseeded Ge NWs.

For our study we chose heavily doped self-seeded Ge NWs predominantly having the same crystallographic direction $[11,14,15]$. Individual NWs were transferred onto $300 \mathrm{~nm}$ thermally grown $\mathrm{SiO}_{2}$ substrates and contacted lithographically in four-point probe configuration [11]. Electrical characterization was carried out at ambient conditions. The geometry of each NW device (diameter size and channel length) was determined by electron microscopy $[11,16]$. From this the NW resistivity was extracted as function of diameter (Figure 1).

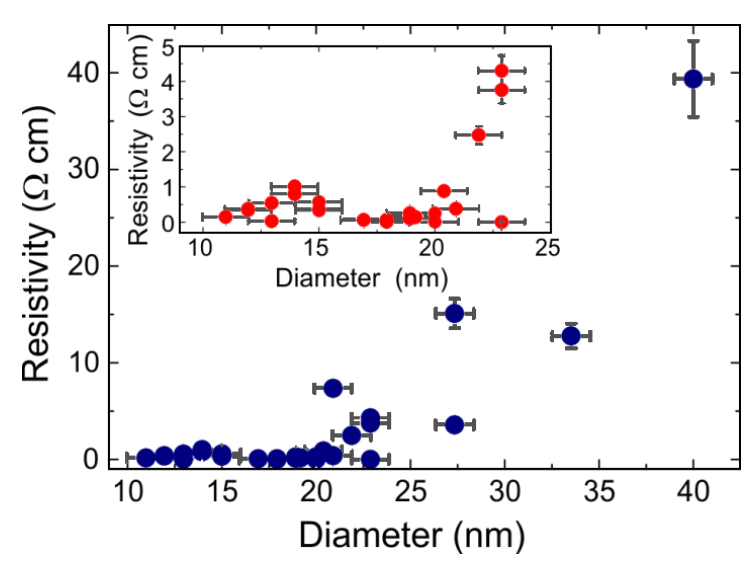

Figure 1: Resistivity of nanowires with 40 to $11 \mathrm{~nm}$ diameter. The resistivity decreases by two orders of magnitude with reducing diameter. In the range of 27 to $20 \mathrm{~nm}$ an increased scattering of the resistivity values is observed. Below $20 \mathrm{~nm}$ the resistivity variation is significantly smaller. Inset: Data for NWs with diameter below $25 \mathrm{~nm}$. At around $14 \mathrm{~nm}$ a peak-like feature is found.

Upon NW diameter reduction, first the resistivity revealed a rapid drop by two orders of magnitude, followed by a region of weakly varying resistivity below $20 \mathrm{~nm}$ diameter. Prior to this region, an increased scattering of the resistivity values was observed coinciding with the NW diameter reaching the excitonic Bohr radius ( $\approx 24 \mathrm{~nm}[10])$, indicating the approach to the quantum regime. Furthermore, a peak-like feature was found at around $14 \mathrm{~nm}$ diameter (inset Figure 1). In comparison, lightly doped semiconducting NWs were reported to exhibit a strong increase of resistivity with decreasing diameter [17].
To describe our findings, we first recall that in self-seeded Ge NWs the majority charge carriers are free holes whose concentration depends on the number of charge traps at the NW core/ shell interface $[11,18]$. In particular, for larger diameter NWs those free holes will be predominantly located in a space-charge region of width $d$ near the interface [19-21] which extends towards the NW centre (schematic in Figure 2a). That is, there will be a central region along the NW axis devoid of free charge carriers. The width $d$ can be calculated by solving the Poisson equation in cylindrical coordinates [20,21] and for simplicity assuming a constant free-hole concentration $n_{\mathrm{h}}$. One finds the expression [22]

$$
-\frac{2 \pi \varepsilon_{0} \varepsilon_{\mathrm{r}} \Phi_{0}}{e n_{\mathrm{h}}}=R^{2}\left(1-\frac{d}{R}\right)^{2} \cdot\left(1-2 \ln \left(1-\frac{d}{R}\right)\right)-R^{2}
$$

where $\Phi_{0}$ is the electrostatic potential at the core/shell interface, $\varepsilon_{0}$ is the vacuum permittivity, $\varepsilon_{\mathrm{r}}$ the dielectric constant of germanium, and $e$ the elementary charge.

a
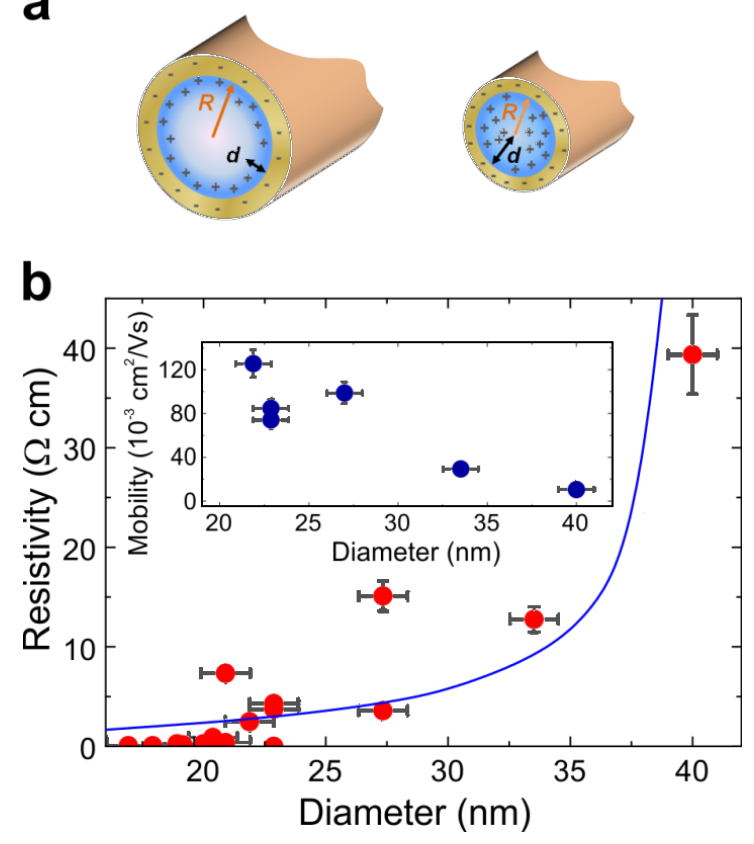

Figure 2: (a) Schematic diagram of the volume in which holes are predominantly located for (left) a wire with a diameter above $\approx 20 \mathrm{~nm}$ and (right) for smaller diameters. The yellow region at the outside represents the nanowire shell. Dark-blue area: region of free holes near to the core/shell interface. Light-coloured area: region devoid of free holes. Trapped electrons and free holes are schematically depicted by $(-)$ and $(+)$, respectively. (b) Comparison between theoretical model and experimental resistivity values for diameters larger than $22 \mathrm{~nm}$. The model (blue line) follows qualitatively the observed experimental data. For smaller diameters a systematic deviation of the data is found indicating the limit of the validity of the model for these diameters. Inset: Calculated mobility values after Equation 2. 
The confinement of free holes into the space-charge region close to the NW surface only, however, cannot remain for all diameters. The available volume for the free holes and the number of charge-traps at the core/shell interface scale with NW diameter. Therefore, with decreasing diameter the holes will extend further towards the NW axis and eventually $d$ will become comparable to the NW radius $R$ which we find in our calculation to occur for a diameter of about $18 \mathrm{~nm}$ (Supporting Information File 1).

We first address the data in the region with the largest resistivity variation (diameters $\geq 20 \mathrm{~nm}$ ). Here we presume $d<<R$ and a classical description to be sufficient. Assuming that the number of holes is the same as the number of charge traps, we get for the free-hole concentration $n_{\mathrm{h}}=2 \eta_{\mathrm{ct}} R \cdot\left(2 R d-d^{2}\right)^{-1}$ with $\eta_{\mathrm{ct}}$ being the charge trap density per $\mathrm{cm}^{2}$. The NW resistivity can be therefore written as (Supporting Information File 1)

$$
\rho_{d<<R}=\left(2 R d-d^{2}\right) \cdot\left(2 e \eta_{\mathrm{ct}} R \mu\right)^{-1}
$$

where $\mu$ is the free hole mobility. Taking $\Phi_{0}=0.3 \mathrm{eV}$ [22], $\varepsilon_{\mathrm{r}}=16$ [23] and $\eta_{\mathrm{ct}}=10^{13} \mathrm{~cm}^{-2}$ [11] we can calculate from Equation 2 the corresponding mobility values (inset Figure $2 b$ ) which range from 10 to $120 \times 10^{-3} \mathrm{~cm}^{2} \mathrm{~V}^{-1} \mathrm{~s}^{-1}$. Interestingly, the mobility increases with reducing diameter, in contrast to lightly doped semiconductor NWs [17].

Fitting the mobility data by a linear function and inserting it into Equation 2 we find resistivity values which are in good agreement with our data for NWs down to $22 \mathrm{~nm}$ diameter (Figure 2b) (showing consistency with our assumption $d<<R$ for nanowires $\geq 18 \mathrm{~nm}$ diameter; Supporting Information File 1). Below $22 \mathrm{~nm}$, however, the resistivity values systematically stay well beneath our theoretical curve indicating that a classical description is insufficient. This is further corroborated by the observation of an increased scattering of the resistivity values around $22 \mathrm{~nm}$ (cf. Figure 1). Both findings point towards a crossover from a classical to a quantum-determined behaviour of the charge-carriers in this diameter range. Therefore, for NW diameters below $20 \mathrm{~nm}$ we now employ a quantum formalism taking into account that the free holes are distributed across the entire NW diameter $(d=R)$. We consider the simple model of a NW being represented by a cylindrical well with infinite potential walls. It is shown below that despite this simplification we find qualitative correspondence with our experimental findings.

Within this approach using the parameters of bulk germanium we determined the positions of the heavy and light hole sub- band minima along with the corresponding density of states (Supporting Information File 1). The density of states for heavy holes was found to dominate and therefore these can be considered as the main carrier type. The average sub-band bottom spacing compares to the thermal energy at room temperature (Supporting Information File 1) suggesting that a description within the 1D Kubo-Greenwood framework is valid [24]. These findings also demonstrate that indeed at diameters $\leq 20 \mathrm{~nm}$ the confinement is not negligible and therefore the quantum regime is entered.

As dominating resistivity contributions, we considered phonon and (remote) Coulomb scattering from the charge traps at the core/shell interface (Supporting Information File 1). Surface roughness scattering was neglected within our diameter range, as previous reports on Si NWs suggest that only at diameters below $10 \mathrm{~nm}$ a sizable contribution is expected $[24,25]$. We further assumed the density of surface states to be constant.

For diameters between 11 and $22 \mathrm{~nm}$, the NW resistivity and mobility values were calculated (Figure 3 ). A peak feature in resistivity at around $14 \mathrm{~nm}$ was found, in similarity to the experimental data (inset Figure 3a). The calculated mobility values decrease monotonically, developing a plateau between 12 and $15 \mathrm{~nm}$ diameter. This can be related to the fact that the Fermi level rises rapidly with these diameters as it is pinned by the large density of states at the bottom of sub-bands involved (Supporting Information File 1) and thus is responsible for the peak feature: The carrier concentration increases with diameter reduction (acting to lower the resistivity), in opposition to the decreasing mobility. That is, when the mobility is constant, the concentration increase dominates and the resistivity reduces as the diameter is lowered. For other diameters the mobility reduction counterbalances the concentration increase leading to a resistivity augmentation with decreasing diameter. Therefore the change from mobility- to carrier-concentration-dominated resistivity leads to the peak-feature observed. Also, the appearance of such a peak is only expected in thin nanowires (sufficiently confined electronic system) with significant interface/ surface state doping.

Although the position of the peak-like feature at $14 \mathrm{~nm}$ is well qualitatively reproduced, there is less quantitative agreement: For the lowest value of $9 \times 10^{-3} \Omega \mathrm{cm}$ for a $19 \mathrm{~nm}$ diameter $\mathrm{NW}$, the estimated $3.4 \times 10^{-3} \Omega \mathrm{cm}$ differs only by a factor of three. However, for the top of the peak feature, the calculated $4.2 \times 10^{-3} \Omega \mathrm{cm}$ is underestimated by more than two orders of magnitude. We attribute this discrepancy to the simplifications used. The infinite potential well approximation (rather than a self-consistent solution to the Schrödinger and Poisson equa- 


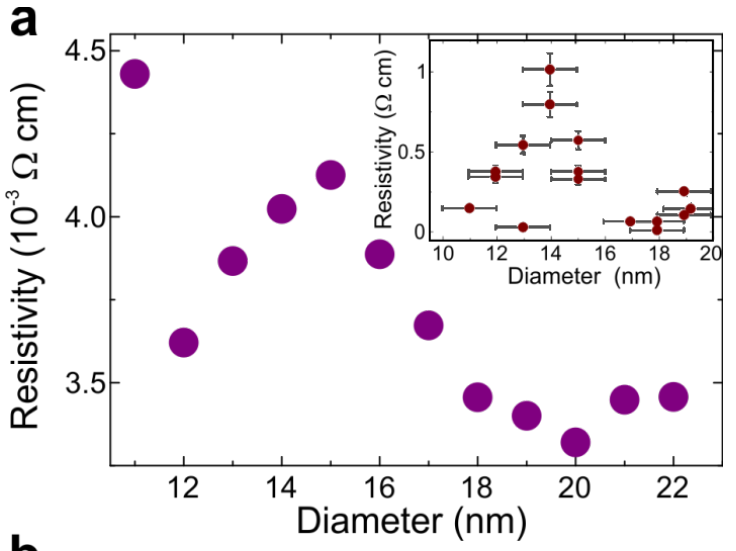

b

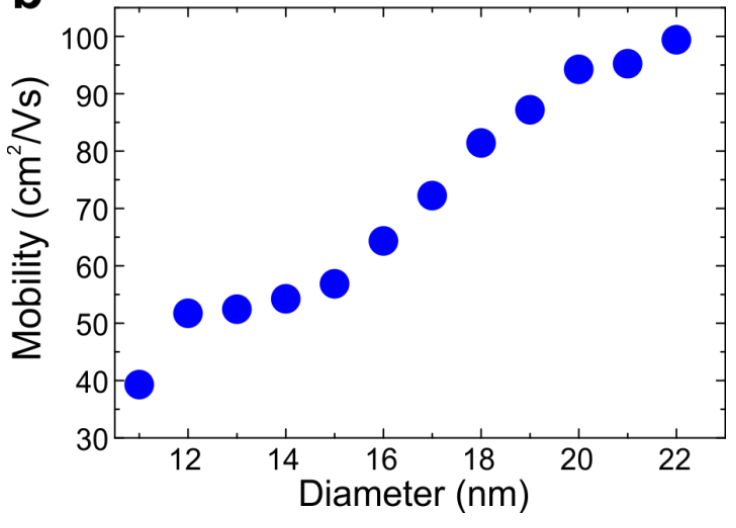

Figure 3: (a) Calculated nanowire resistivity from 22 to $11 \mathrm{~nm}$ diameter. Around $14 \mathrm{~nm}$ a peak-feature is found in agreement with the experimental data. At $11 \mathrm{~nm}$ diameter, the sharp increase of the resistivity is due to the shift of the Fermi-energy below the third heavy-hole sub-band (Supporting Information File 1). (b) Nanowire mobility calculated for the same diameter range as in (a). After an initial decrease, the mobility reveals a plateau-like region from about 12 to $15 \mathrm{~nm}$.

tions) requires the carrier wavefunctions to be strictly zero at the cylinder surface which in turn results in an underestimate of the charge carrier density within the NW surface region. This influences the actual Coulomb-scattering contribution to the resistivity as the Coulomb-scattering amplitude between any two sub-bands is strongly dependent on the NW diameter (Supporting Information File 1). While for all diameters the scattering amplitude principally increases when approaching the core/shell interface, within larger diameter NWs the difference for carriers in the centre and close to the surface augments significantly. More specifically, this increase is predominantly due to the rapid change of the scattering amplitude in the NW centre, while close to the surface the amplitude varies much less for different diameters. For example, the scattering amplitude within the centre region of a $12 \mathrm{~nm}$ diameter nanowire is more than an order of magnitude higher compared to a NW of $17 \mathrm{~nm}$ diameter. In contrast, the amplitudes close to the NW surfaces differ only by a few ten \% (Supporting Information File 1).

\section{Supporting Information}

\section{Supporting Information File 1}

Details on theoretical calculations.

[http://www.beilstein-journals.org/bjnano/content/

supplementary/2190-4286-7-119-S1.pdf]

\section{Acknowledgements}

This work was supported by the Science Foundation Ireland contract-number PI-award 08/IN.1/I1873 and in part by CSET 08/CE/I1432.

\section{References}

1. Xiang, J.; Lu, W.; Hu, Y.; Wu, Y.; Yan, H.; Lieber, C. M. Nature 2006, 441, 489-493. doi:10.1038/nature04796

2. Wang, D.; Wang, Q.; Javey, A.; Tu, R.; Dai, H.; Kim, H.; McIntyre, P. C.; Krishnamohan, T.; Saraswat, K. C. Appl. Phys. Lett. 2003, 83, 2432-2434. doi:10.1063/1.1611644

3. Zhang, L.; Tu, R.; Dai, H. Nano Lett. 2006, 6, 2785-2789. doi:10.1021/nl061833b

4. Zheng, G.; Lu, W.; Jin, S.; Lieber, C. M. Adv. Mater. 2004, 16, 1890-1893. doi:10.1002/adma.200400472

5. Liang, G.; Xiang, J.; Kharche, N.; Klimeck, G.; Lieber, C. M.; Lundstrom, M. Nano Lett. 2007, 7, 642-646. doi:10.1021/nl062596f

6. Li, Y.; Qian, F.; Xiang, J.; Lieber, C. M. Mater. Today 2006, 9, 18-27. doi:10.1016/S1369-7021(06)71650-9

7. Goldberger, J.; Hochbaum, A. I.; Fan, R.; Yang, P. Nano Lett. 2006, 6, 973-977. doi:10.1021/nl060166j

8. Lieber, C. M. MRS Bull. 2003, 28, 486-491. doi:10.1557/mrs2003.144

9. Lu, W.; Lieber, C. M. Nat. Mater. 2007, 6, 841-850. doi:10.1038/nmat2028

10. Gu, G.; Burghard, M.; Kim, G. T.; Düsberg, G. S.; Chiu, P. W.; Krstić, V.; Roth, S.; Han, W. Q. J. Appl. Phys. 2001, 90, 5747-5751. doi:10.1063/1.1413495

11. Connaughton, S.; Hobbs, R.; Lotty, O.; Holmes, J. D.; Krstić, V. Adv. Mater. Interfaces 2015, 2, 1400469. doi:10.1002/admi.201400469

12. Barth, S.; Koleśnik, M. M.; Donegan, K.; Krstić, V.; Holmes, J. D. Chem. Mater. 2011, 23, 3335-3340. doi:10.1021/cm200646e

13. Collins, G.; Koleśnik, M.; Krstić, V.; Holmes, J. D. Chem. Mater. 2010, 22, 5235-5243. doi:10.1021/cm1012137

14. Hobbs, R. G.; Barth, S.; Petkov, N.; Zirngast, M.; Marschner, C.; Morris, M. A.; Holmes, J. D. J. Am. Chem. Soc. 2010, 132, 13742-13749. doi:10.1021/ja1035368

15. Lotty, O.; Hobbs, R.; O’Regan, C.; Hlina, J.; Marschner, C.; O'Dwyer, C.; Petkov, N.; Holmes, J. D. Chem. Mater. 2013, 25, 215-222. doi:10.1021/cm3032863

16. Koleśnik-Gray, M. M.; Lutz, T.; Collins, G.; Biswas, S.; Holmes, J. D.; Krstić, V. Appl. Phys. Lett. 2013, 103, 153101. doi:10.1063/1.4821996

17. Scheffler, M.; Nadj-Perge, S.; Kouwenhoven, L. P.; Borgström, M. T.; Bakkers, E. P. A. M. J. Appl. Phys. 2009, 106, 124303. doi:10.1063/1.3270259

18. Xie, P.; Hu, Y.; Fang, Y.; Huang, J.; Lieber, C. M. Proc. Natl. Acad. Sci. U. S. A. 2009, 106, 15254-15258. doi:10.1073/pnas.0906943106

19. Bardeen, J. Phys. Rev. 1947, 71, 717-727. doi:10.1103/PhysRev.71.717 
20. Cardona, M.; Yu, P. Y. Fundamentals of semiconductors; Springer: Berlin, Germany, 2005. doi:10.1007/b137661

21. Fahad, H. M.; Hussain, M. M. Sci. Rep. 2012, 2, No. 475. doi:10.1038/srep00475

22. Wang, D.; Chang, Y.-L.; Wang, Q.; Cao, J.; Farmer, D. B.; Gordon, R. G.; Dai, H. J. Am. Chem. Soc. 2004, 126, 11602-11611. doi:10.1021/ja047435x

23. Young, K. F.; Frederikse, H. P. R. J. Phys. Chem. Ref. Data 1973, 2, 313-409. doi:10.1063/1.3253121

24. Jin, S.; Fischetti, M. V.; Tang, T.-w. Appl. Phys. Lett. 2007, 102, 083715. doi:10.1063/1.2802586

25. Dura, J.; Triozon, F.; Barraud, S.; Munteanu, D.; Martinie, S.; Autran, J. L. J. Appl. Phys. 2012, 111, 103710-103719. doi:10.1063/1.4719081

\section{License and Terms}

This is an Open Access article under the terms of the Creative Commons Attribution License

(http://creativecommons.org/licenses/by/4.0), which permits unrestricted use, distribution, and reproduction in any medium, provided the original work is properly cited.

The license is subject to the Beilstein Journal of

Nanotechnology terms and conditions:

(http://www.beilstein-journals.org/bjnano)

The definitive version of this article is the electronic one which can be found at: doi:10.3762/bjnano.7.119 